\title{
Generation and Evolution of Chaos in Double-Well Duffing Oscillator under Parametrical Excitation
}

\author{
Ying Zhang, ${ }^{1}$ Xiaole Yue, ${ }^{1}$ Lin Du, ${ }^{1}$ Liang Wang, ${ }^{1}$ and Tong Fang ${ }^{2}$ \\ ${ }^{1}$ Department of Applied Mathematics, Northwestern Polytechnical University, Xian 710072, China \\ ${ }^{2}$ Department of Engineering Mechanics, Northwestern Polytechnical University, Xian 710072, China \\ Correspondence should be addressed to Ying Zhang; yingzhang1031@nwpu.edu.cn
}

Received 24 June 2016; Accepted 4 August 2016

Academic Editor: Tai Thai

Copyright (C) 2016 Ying Zhang et al. This is an open access article distributed under the Creative Commons Attribution License, which permits unrestricted use, distribution, and reproduction in any medium, provided the original work is properly cited.

\begin{abstract}
The generation and evolution of chaotic motion in double-well Duffing oscillator under harmonic parametrical excitation are investigated. Firstly, the complex dynamical behaviors are studied by applying multibifurcation diagram and Poincaré sections. Secondly, by means of Melnikov's approach, the threshold value of parameter $\mu$ for generation of chaotic behavior in Smale horseshoe sense is calculated. By the numerical simulation, it is obvious that as $\mu$ exceeds this threshold value, the behavior of Duffing oscillator is still steady-state periodic but the transient motion is chaotic; until the top Lyapunov exponent turns to positive, the motion of system turns to permanent chaos. Therefore, in order to gain an insight into the evolution of chaotic behavior after $\mu$ passing the threshold value, the transient motion, basin of attraction, and basin boundary are also investigated.
\end{abstract}

\section{Introduction}

Duffing oscillator, named after Georg Duffing, is a famous damped and forced nonlinear dynamical system [1]. From the last century, the Duffing-type nonlinear dynamical systems have been investigated uninterruptedly in so many fields by plenty of researches, such as physics, engineering, chemistry, economics, and biological and social sciences [2-5]. Moreover, it is famous for the existence of chaos behavior in recent decades. In 1979, the chaotic phenomena in Duffing's equation had been investigated by Ueda [6]. In his research, by using the numerical simulation, the changes of attractors were obtained under the various parameters. From then on, the chaotic behavior in Duffing's equation under a harmonic excitation is known as a common dynamical phenomenon. Furthermore, the crisis which is a kind of bifurcation of chaotic behavior is studied in many literatures [7-9], and it had been defined and analyzed generally by Ott published in [10]. In recent years, scientists are still concerned about the problems in Duffing-type systems, for example, suppressing and inducing of chaos, influence of time delay, fractional dynamics, and stochastic dynamics [11-15]. In our previous work [16, 17], the bifurcation and crises had been explored deeply in double-well Duffing system under harmonic parametric excitation, to exhibit the transformations between different dynamical behaviors. However, the generation of chaos was not mentioned, and it is really important in theory and practice.

Melnikov method is a significant analytical approach to giving the criterion of the existence of chaotic behavior, which is introduced by Melnikov in 1963 [18] and investigated intensively in [19] by Guckenheimer and Holmes. The main idea of this method is to measure the distance between the stable and unstable manifolds. If the stable and unstable manifolds intensively intersect once, they will intersect infinite times. Thus, according to Smale-Birkhoff theorem [19], it implies the existence of the chaotic behavior in Smale horseshoe sense. The Melnikov theory was firstly used to study chaos in Duffing system by Holmes [20], and generalized Melnikov function is developed by Wiggins [21, 22]. This criterion is just the necessary condition but not sufficient condition for chaos; therefore, it must be the sufficient conditions for the suppression of chaos [23]. 


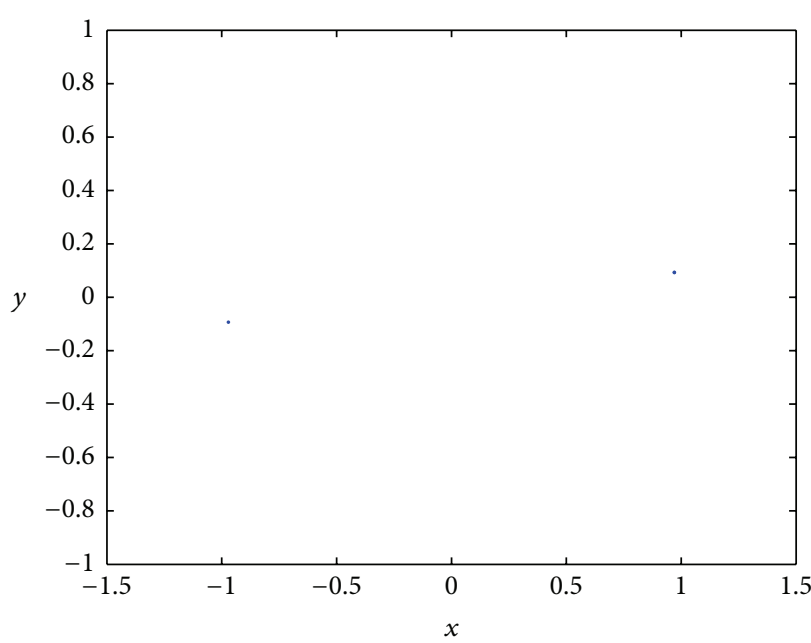

(a)

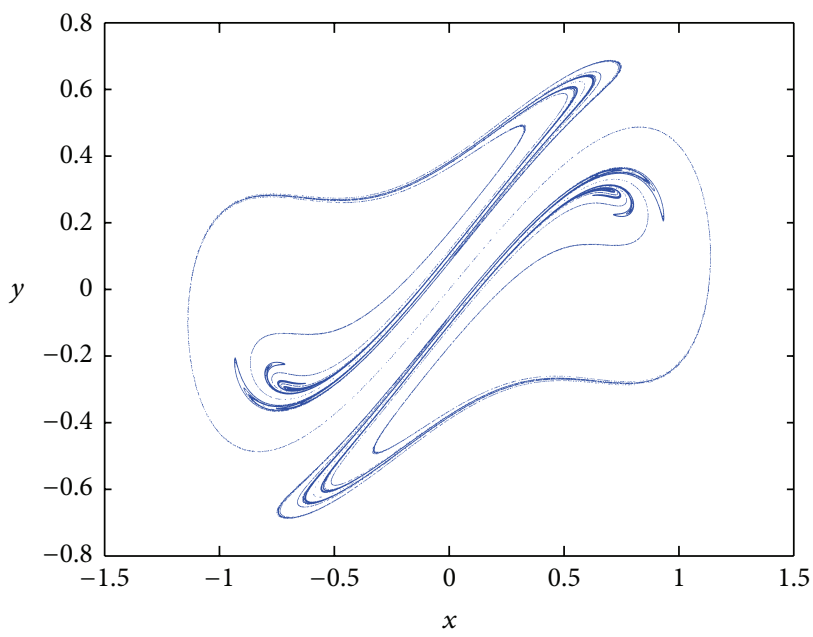

(c)

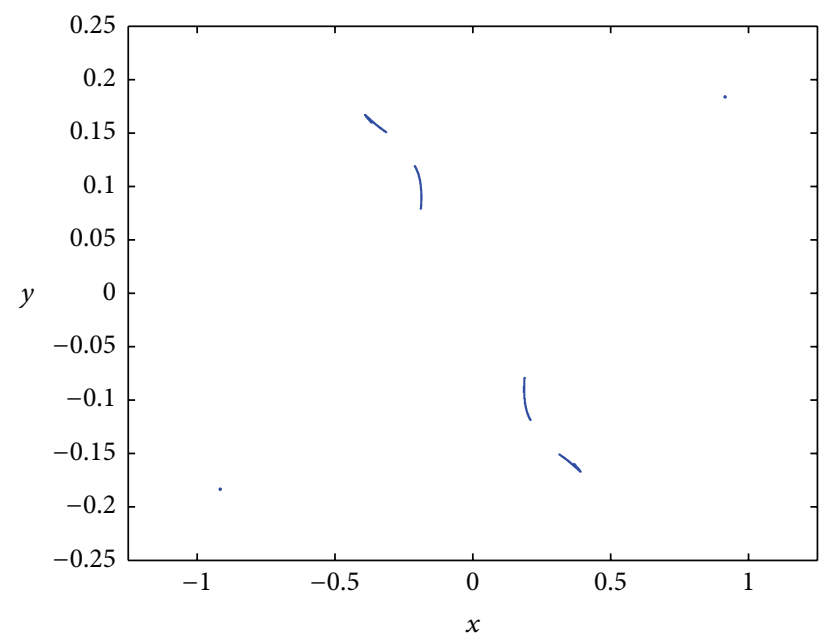

(b)

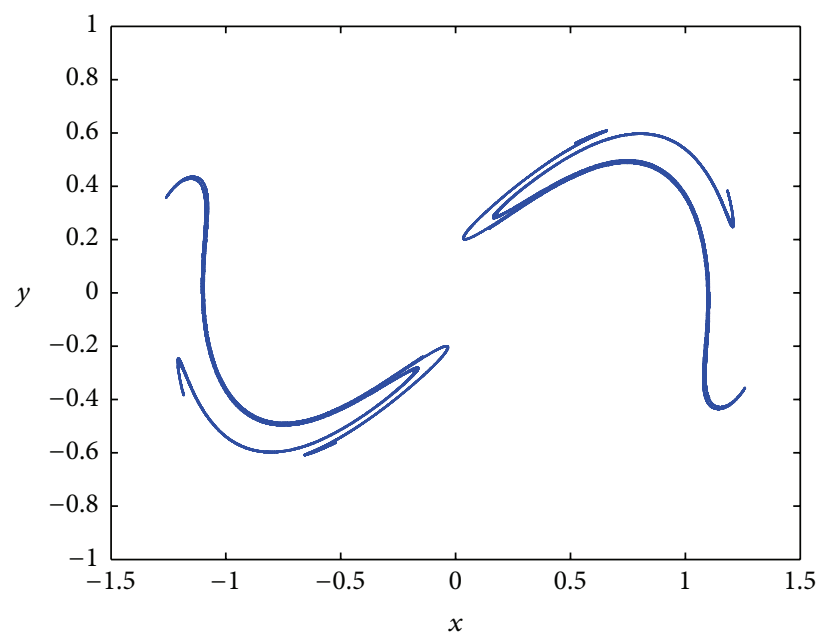

(d)

Figure 1: Poincaré sections of system (2) for (a) $\mu=0.10$, (b) $\mu=0.205$, (c) $\mu=0.35$, and (d) $\mu=0.90$.

In this paper, we mainly investigate the occurrence of the chaotic behaviors in double-well Duffing system forced by parametrical excitation and explore the dynamical phenomena between the occurrences of horseshoe chaos and permanent chaos. The rest part of this paper is organized as follows. In Section 2, the dynamical behaviors in a large parameter range is studied. Section 3 gives the threshold curves for the generation of Smale horseshoe chaos by Melnikov function. In Section 4, the dynamical behaviors after the intersection of manifolds and before the occurrence of permanent chaos are investigated. Finally, some conclusions are given in Section 5.

\section{Dynamical Behaviors in Double-Well Duffing System}

The double-well Duffing system under a harmonic parametrical excitation is shown as the following:

$$
\ddot{x}+\varepsilon \widehat{\varepsilon} \dot{x}-a x+b x^{3}=\varepsilon \widehat{\mu} x \sin \Omega t,
$$

where $a, b$, and $\widehat{c}$ are positive coefficients, $\widehat{\mu}$ and $\Omega$ are the amplitude and frequency of the excitation, and $0<\varepsilon \ll 1$. By denoting $\dot{x}=y$, (1) can be rewritten as the following form:

$$
\begin{aligned}
& \dot{x}=y, \\
& \dot{y}=a x-b x^{3}-\varepsilon \widehat{c} y+\varepsilon \widehat{\mu} x \sin \Omega t .
\end{aligned}
$$

It is clear that Duffing system (2) is symmetrical to origin $(0,0)$ as shown in Figure 1. The chaotic attractor in Figure 1(c) is self-symmetrical, and the coexisting attractors are all symmetrical with each other in Figures 1(a), 1(b), and 1(d). Letting $c=\varepsilon \widehat{\varepsilon}$ and $\mu=\varepsilon \widehat{\mu}$ in system (2), when denoting $\mu=0.10$, Duffing system keeps two $1 T$ periodic attractors symmetrical with each other; for $\mu=0.35$ or $\mu=0.90$, only chaotic attractor exists but with one or two branches; and, for $\mu=0.205$, two $1 T$ periodic attractors and two chaotic attractors coexist. The changes between different symmetrical forms are all result from the bifurcations and crises.

In our previous work, plenty of interesting dynamical behaviors have been investigated within $\mu \in[4.9,7.2]$, such as 


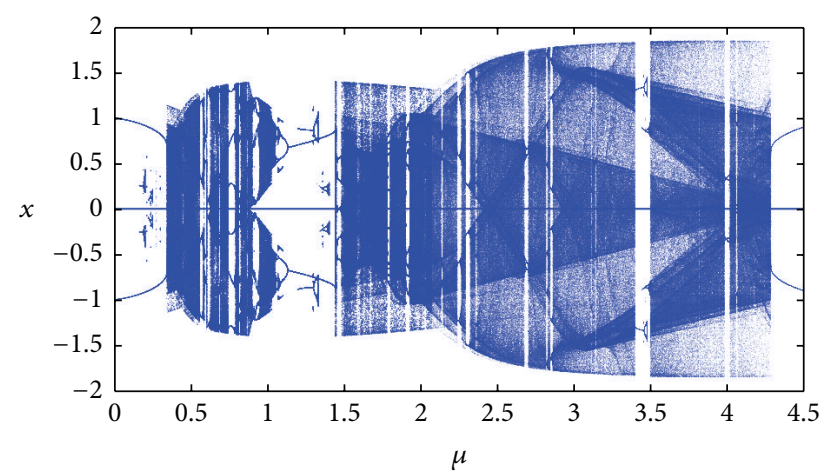

Figure 2: Multibifurcation diagram of system (2) when $\mu \in(0,4.5]$.

period doubling bifurcation cascades, chaos crises, periodic windows, chaotic saddles, fractal boundary of basin, and transition chaos. In this paper, from Figure 2, we can see that the dynamics are much more complicated as shown in the multibifurcation diagram. The "multi" we used means that the data on this bifurcation diagram is simulated from a certain quantity of initial condition (IC), to make sure that all the coexisting attractors under the same parameter values can be presented.

\section{Melnikov's Approach}

Based on our previous work in [16, 17], it is shown that if the system is once chaotic, then the behavior of this system will always bifurcate between chaotic attractors and chaotic saddles. This means that the system will not go back to regular motions with the increasing of $\mu$ and keep on bifurcating between permanent chaos and transient chaos. Therefore, in order to gain insight into the evolution of chaotic dynamical behaviors, it is essential to obtain the sufficient conditions for the generation of chaos. Thus, Melnikov's approach and the top Lyapunov exponents (TLE) $[24,25]$ are proposed to calculate the threshold values for chaotic motions in Duffing system (2).

If we let $\varepsilon=0$, the unperturbed system can be written as

$$
\begin{aligned}
& \dot{x}=y, \\
& \dot{y}=a x-b x^{3},
\end{aligned}
$$

which is a Hamiltonian system, and the Hamiltonian function is

$$
H(x, y)=\frac{1}{2} y^{2}-\frac{a}{2} x^{2}+\frac{b}{4} x^{4}=h
$$

with the corresponding potential function

$$
V(x)=-\frac{a}{2} x^{2}+\frac{b}{4} x^{4}
$$

The curve of function (5) has two symmetrical wells separated by the barrier at $x=0$ shown in Figure 3(a), such that system (2) is called double-well Duffing system. In the unperturbed system (3), we can find that there exist three equilibrium points: hyperbolic saddle $(0,0)$ and two symmetrical center points $( \pm \sqrt{a / b}, 0)$ shown in Figure 3(b). Meanwhile, around the centers $( \pm \sqrt{a / b}, 0)$, there are two symmetrical homoclinic orbits (6) beginning and ending at the hyperbolic saddle $(0,0)$ as $t \rightarrow \infty$ drawn in Figure 3(b):

$$
\begin{aligned}
& x_{0}(t)= \pm \sqrt{\frac{2 a}{b}} \operatorname{sech}(\sqrt{a} t), \\
& y_{0}(t)=\mp \sqrt{\frac{2 a}{b}} \sqrt{a} \tanh (\sqrt{a} t) \operatorname{sech}(\sqrt{a} t) .
\end{aligned}
$$

Based on the Smale-Birkhoff theorem [19], it is well know that once a stable manifold and an unstable manifold transversally intersect, the chaos will appear in the sense of Smale horseshoe. In order to get the parameter regions for chaotic motions, Melnikov's approach, which is an analytical and quantificational method, is applied in this section. This method is to measure the distance between stable manifolds and unstable manifolds of the saddle in unperturbed system. If the Melnikov function has a simple zero, the stable and unstable manifolds will transversally intersect infinite times. Therefore, in order to investigate the occurrence of chaotic behavior and get the threshold value of parameters, the simple zero of Melnikov function should be solved.

The Melnikov function of system (2) is shown as

$$
\begin{aligned}
M( & \left.t_{0}\right) \\
= & \int_{-\infty}^{+\infty} y_{0}(t)\left[-\widehat{c} y_{0}(t)+\widehat{\mu} x_{0}(t) \sin \Omega\left(t+t_{0}\right)\right] d t \\
= & \int_{-\infty}^{+\infty}-\widehat{c}\left(y_{0}(t)\right)^{2} d t \\
& +\int_{-\infty}^{+\infty} \widehat{\mu} x_{0}(t) y_{0}(t) \sin \Omega\left(t+t_{0}\right) d t \\
= & -\widehat{c} I_{1}+\widehat{\mu} I_{2}\left(t_{0}\right) .
\end{aligned}
$$

Then, taking the function of homoclinic orbits (6) into $I_{1}$ and $I_{2}$, we can get

$$
\begin{aligned}
& I_{1}=\int_{-\infty}^{+\infty}\left(y_{0}(t)\right)^{2} d t=\frac{2 a^{2}}{b} \int_{-\infty}^{+\infty} \tanh ^{2}(\sqrt{a} t) \\
& \cdot \operatorname{sech}^{2}(\sqrt{a} t) d t=\left.\frac{2 a^{2}}{b} \frac{1}{\sqrt{a}} \frac{1}{3} \tanh ^{3}(\sqrt{a} t)\right|_{-\infty} ^{+\infty} \\
& \quad= \frac{4 a \sqrt{a}}{3 b}, \\
& I_{2}\left(t_{0}\right)=\int_{-\infty}^{+\infty} x_{0}(t) y_{0}(t) \sin \Omega\left(t+t_{0}\right) d t=-\frac{2 a \sqrt{a}}{b} \\
& \cdot \int_{-\infty}^{+\infty} \operatorname{sech}^{2}(\sqrt{a} t) \tanh (\sqrt{a} t) \\
& \cdot\left(\sin \Omega t \cos \Omega t_{0}+\cos \Omega t \sin \Omega t_{0}\right) d t=-\frac{2 a \sqrt{a}}{b} \\
& \cdot \cos \Omega t_{0} \int_{-\infty}^{+\infty} \operatorname{sech}^{2}(\sqrt{a} t) \tanh (\sqrt{a} t) \sin \Omega t d t
\end{aligned}
$$




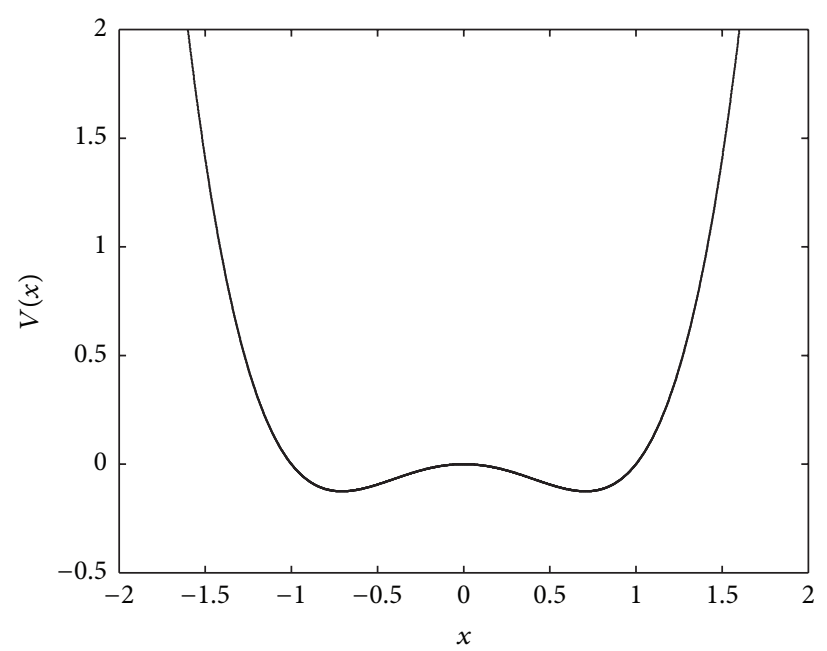

(a)

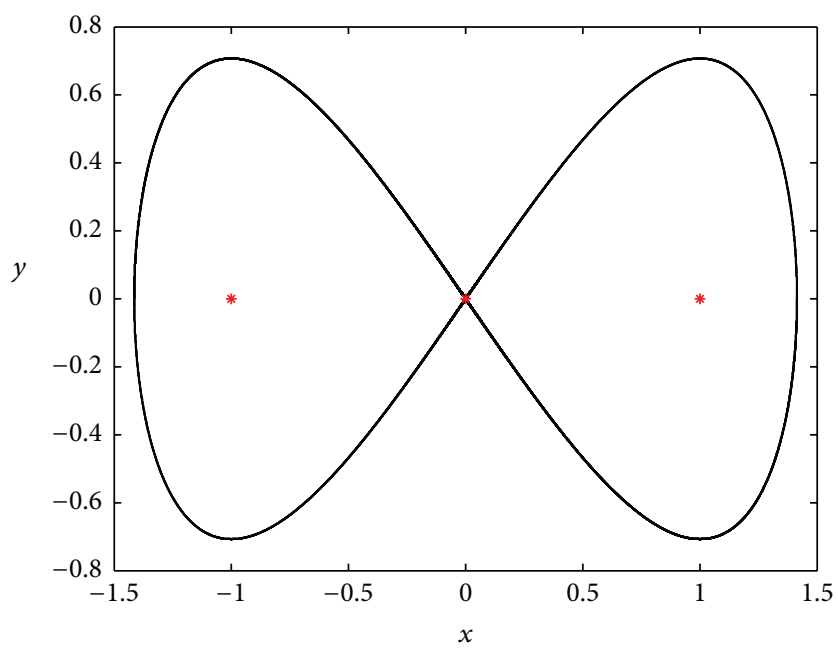

(b)

FIGURE 3: When $a=b=1.0$ in system (3); (a) potential function; (b) two homoclinic orbits (curve) and three equilibrium points (asterisks).

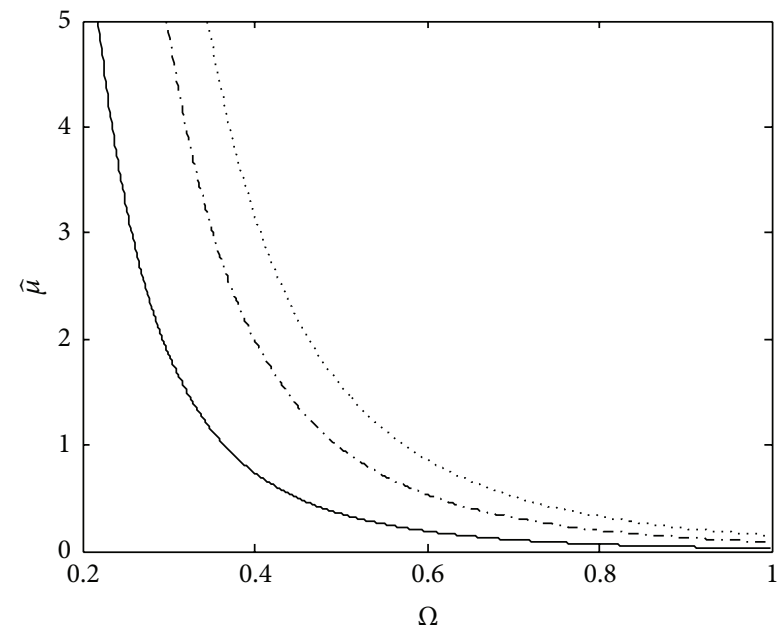

$$
\begin{aligned}
-a & =0.5 \\
-\cdot- & a=0.8 \\
\cdots \cdots & a=1.0
\end{aligned}
$$

FIGURE 4: For $\widehat{c}=0.2$, the critical curves for chaotic behaviors in sense of Smale horseshoe in $(\Omega, \widehat{\mu})$ plane.

$$
\begin{aligned}
& -\frac{2 a \sqrt{a}}{b} \sin \Omega t_{0} \int_{-\infty}^{+\infty} \operatorname{sech}^{2}(\sqrt{a} t) \tanh (\sqrt{a} t) \\
& \cdot \cos \Omega t d t=-\frac{2 a \sqrt{a}}{b} \cos \Omega t_{0} \int_{-\infty}^{+\infty} \operatorname{sech}^{2}(\sqrt{a} t) \\
& \cdot \tanh (\sqrt{a} t) \sin \Omega t d t+0=-\frac{2 a \sqrt{a}}{b} \\
& \cdot \cos \Omega t_{0} \operatorname{Im}\left(\frac{1}{4 a^{3 / 2}} \pi \operatorname{csch}\left(\frac{\pi}{4 \sqrt{a}}\right) \operatorname{sech}\left(\frac{\pi}{4 \sqrt{a}}\right) i\right) \\
& =-\frac{2 a \sqrt{a}}{b} \cdot \frac{\pi \Omega^{2}}{4 a^{3 / 2}} \cdot \operatorname{csch}\left(\frac{\Omega \pi}{4 \sqrt{a}}\right) \operatorname{sech}\left(\frac{\Omega \pi}{4 \sqrt{a}}\right)
\end{aligned}
$$

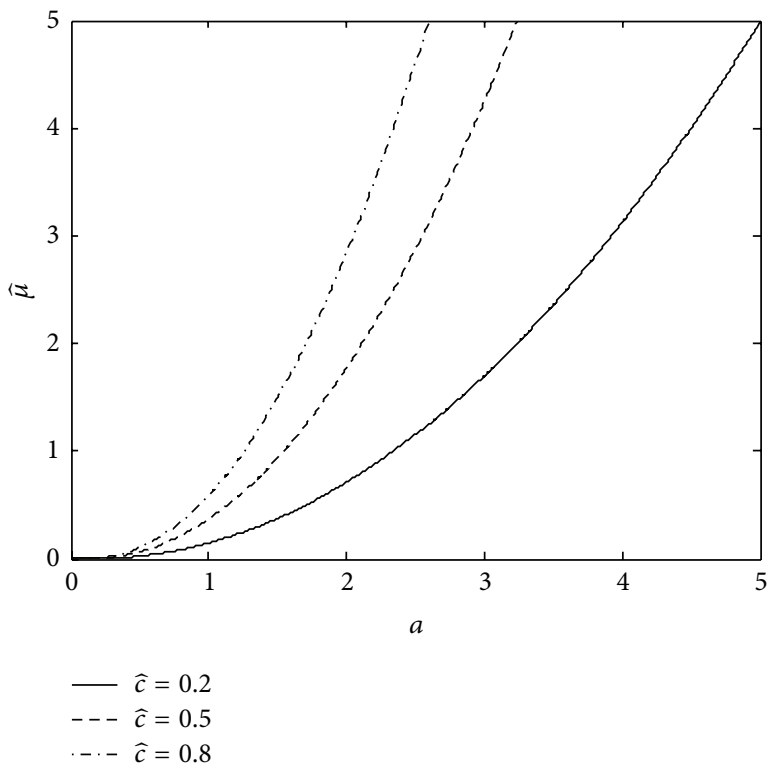

FIGURE 5: For $\Omega=1.0$, the critical curves for chaotic behaviors in sense of Smale horseshoe in $(a, \widehat{\mu})$ plane.

$$
\begin{aligned}
& \cdot \cos \Omega t_{0}=-\frac{\pi \Omega^{2}}{2 b} \cdot \operatorname{csch}\left(\frac{\Omega \pi}{4 \sqrt{a}}\right) \operatorname{sech}\left(\frac{\Omega \pi}{4 \sqrt{a}}\right) \\
& \cdot \cos \Omega t_{0} .
\end{aligned}
$$

After the integrations in (8), the Melnikov function (7) is reduced into the following form:

$$
\begin{aligned}
M\left(t_{0}\right)= & -\frac{4 a \widehat{c} \sqrt{a}}{3 b}-\widehat{\mu} \frac{\pi \Omega^{2}}{2 b} \\
& \cdot \operatorname{csch}\left(\frac{\Omega \pi}{4 \sqrt{a}}\right) \operatorname{sech}\left(\frac{\Omega \pi}{4 \sqrt{a}}\right) \cos \Omega t_{0} .
\end{aligned}
$$




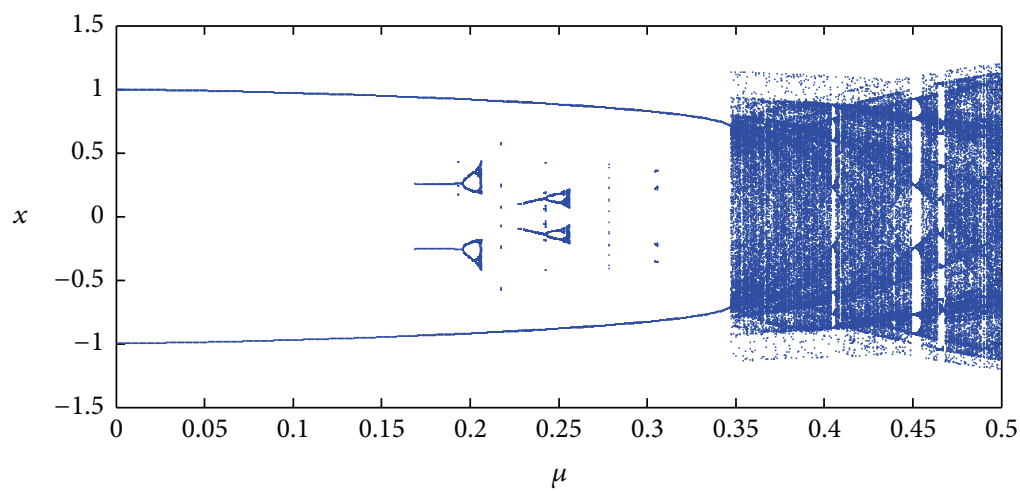

(a)

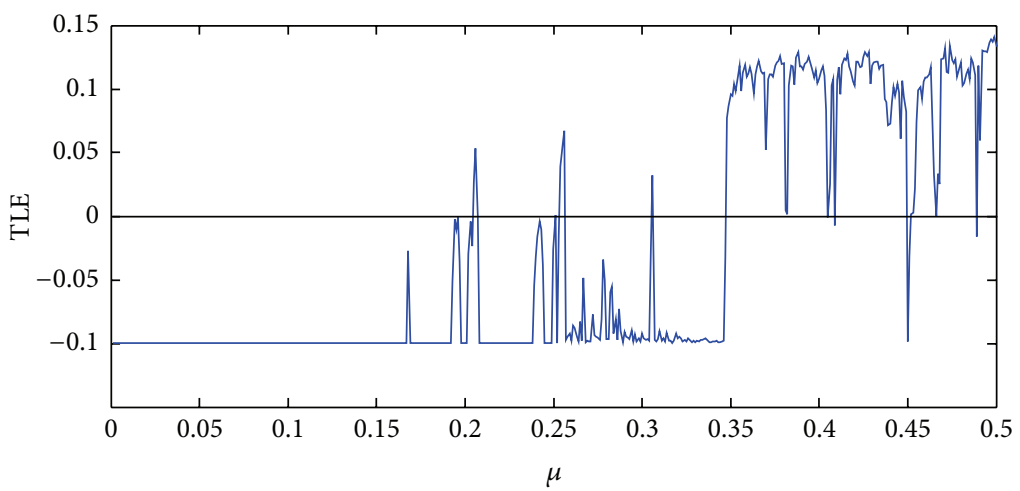

(c)

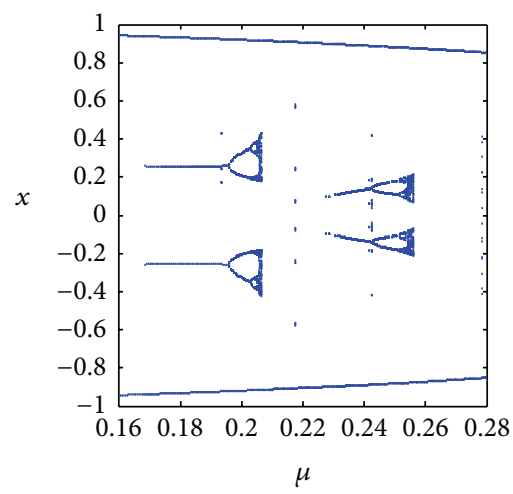

(b)

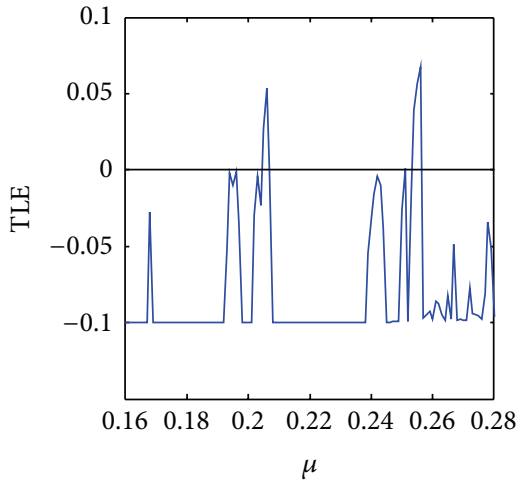

(d)

FIGURE 6: The multibifurcation diagram (a) and multi-TLE diagram (b) of system (2) when $\Omega=1, a=1$, and $c=0.2$, (c) and (d) are zoom in figures for (a) and (b) separately. (The "multi-" in Figure (b) means that, for every certain value of $\mu$, the TLE value is simulated for numbers of different ICs, and the largest one is preserved as the TLE value of this $\mu$.)

In order to get the simple zeros of Melnikov function, we should suppose $M\left(t_{0}\right)=0$ and $M^{\prime}\left(t_{0}\right) \neq 0$. By denoting $M\left(t_{0}\right)=0$, the following identities can be found:

$$
\begin{aligned}
\cos & \Omega t_{0} \\
& =\frac{-4 a \widehat{c} \sqrt{a} / 3 b}{\widehat{\mu}\left(\pi \Omega^{2} / 2 b\right) \cdot \operatorname{csch}(\Omega \pi / 4 \sqrt{a}) \operatorname{sech}(\Omega \pi / 4 \sqrt{a})} \\
& =-\frac{\widehat{c}}{\widehat{\mu}} \cdot \frac{8 a \sqrt{a}}{3 \pi \Omega^{2} \cdot \operatorname{csch}(\Omega \pi / 4 \sqrt{a}) \operatorname{sech}(\Omega \pi / 4 \sqrt{a})},
\end{aligned}
$$

where $\left|\cos \Omega t_{0}\right| \leq 1$. And because $M^{\prime}\left(t_{0}\right) \neq 0$ signifies $\left|\cos \Omega t_{0}\right| \neq 1$, then the necessary and sufficient condition for Melnikov function to have simple zeros is

$$
\begin{aligned}
\frac{\widehat{\mu}}{\widehat{c}} & <\left(\frac{\mu}{c}\right)_{\mathrm{cr}} \\
& =\left|\frac{8 a \sqrt{a}}{3 \pi \Omega^{2} \cdot \operatorname{csch}(\Omega \pi / 4 \sqrt{a}) \operatorname{sech}(\Omega \pi / 4 \sqrt{a})}\right|,
\end{aligned}
$$

where $(\mu / c)_{\mathrm{cr}}$ is the threshold function. It implies that condition (11) guarantees the transversal intersection of stable and unstable manifolds and the possible occurrence of Smale horseshoe chaotic motion. In the following parts of this paper, we aim to investigate the evolution of chaos, by considering threshold condition (11).

In Figure 4, the critical curves for different value of $a$ are drawn in $(\Omega, \widehat{\mu})$ plane. For a certain value of $a$, when the set of parameters are taken above the critical curve, the transversal intersection of stable and unstable manifolds occurs. In addition, the relationship between $\widehat{\mu}, a$, and $\widehat{c}$ can be found in Figure 5, and the Smale horseshoe chaotic behavior will happen in the parameter region above the critical curves too. By analyzing the critical curves, it is evident that the critical value of $\hat{\mu}$ is proportional to $a$ and $\widehat{c}$ but inversely proportional to $\Omega$.

\section{Evaluation of Chaotic Motion}

Based on Melnikov's approach, when $\Omega=1, a=1, b=1$, and $c=0.2$, the threshold value of $\mu$ can be calculated, which is approximately equal to 0.15 . That is to say, when $\mu>0.15$, the stable and unstable manifolds will intersect transversally for infinity times, which implies the generation of chaotic behavior in horseshoe sense. However, in Figure 6, from the multibifurcation and the corresponding multi-TLE diagrams, it is obvious that the steady-state motions of system (2) are not chaotic but periodic when $\mu>0.15$. With the 


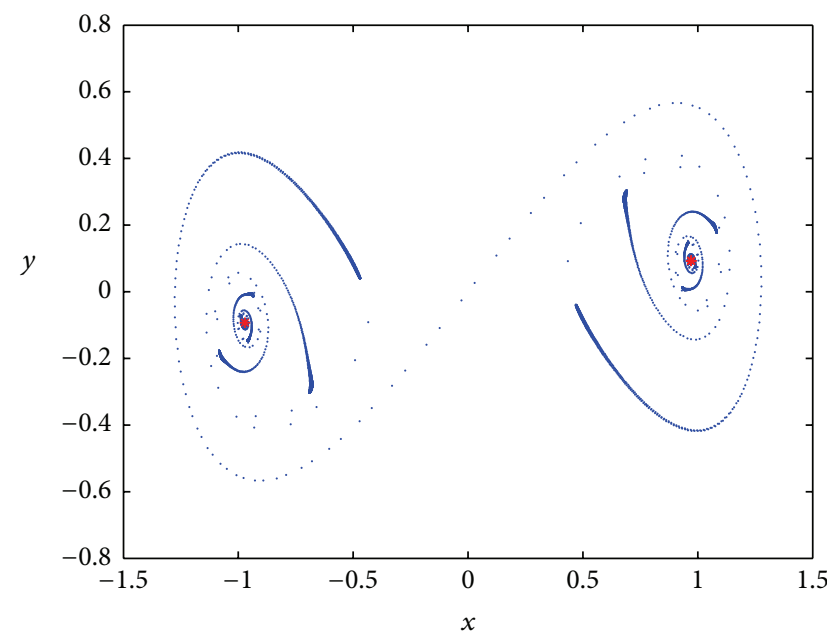

(a)

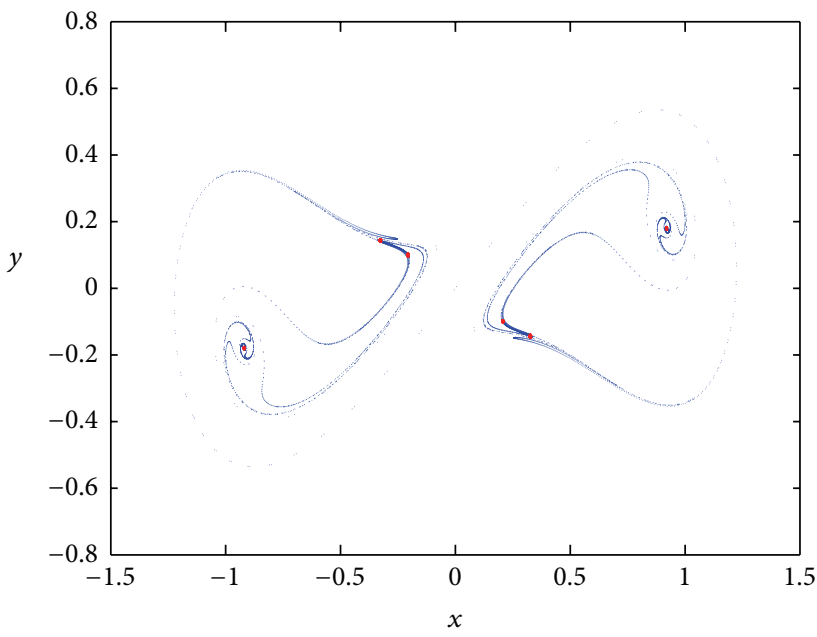

(b)

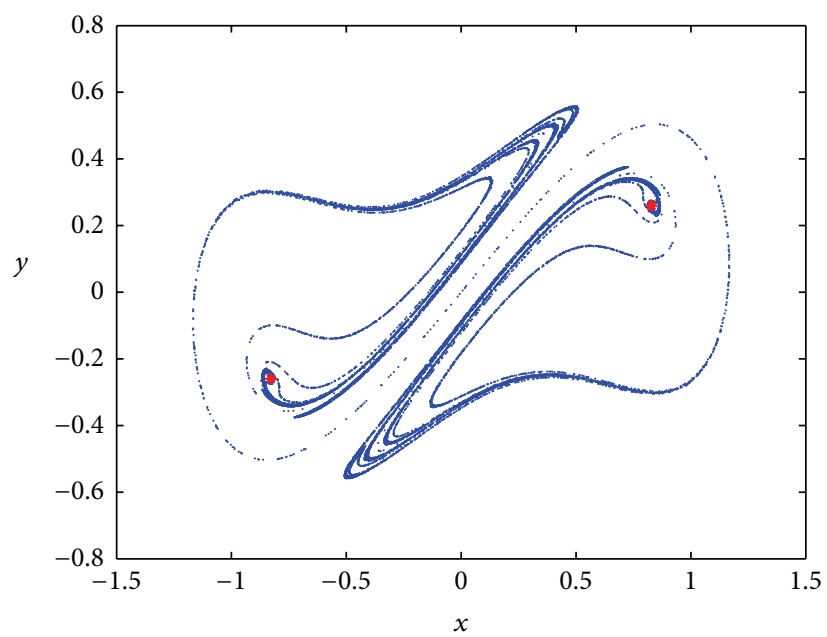

(c)

FIGURE 7: The transient responses (blue dots) and steady attractors (red asterisks) for different values of $\mu$ : (a) $\mu=1.0$, (b) $\mu=2.0$, and (c) $\mu=3.0$.

increasing of parameter $\mu$ and resulting from the double period bifurcation cascade, the permanent chaotic attractors are firstly engendered when $\mu \geq 0.205$ coexisting with two $1 T$ periodic attractors. After that, the behavior of Duffing system (2) is keeping on transforming between permanent chaos and chaotic saddles endlessly via bifurcations and crises [17]. Furthermore, in order to study the evolution of chaos on $\mu \in(0.15,0.205)$, not only the steady states but also the transient behaviors should be investigated in detail.

It is well known that transient chaos and fractal boundary of basin both coexist with chaotic saddle [10], and chaotic attractor and chaotic saddle or transient chaos are the different forms of chaotic behaviors. If the responses of system are captured by the chaotic saddle, they will possibly stay near the saddle for a time chaotically. However, chaotic saddle is nonattracting; eventually, the responses will escape from the saddle and asymptotes to a steady state such as stable periodic orbits, which is called transient chaos. In any case, no matter permanent or transient chaos, they are all described as chaotic, and both play a significant role in theory and reality. It is known that Melnikov criterion is only a necessary condition, just signifying the possibility of occurrence of permanent chaos, which means that this method is an effective approach in giving the necessary condition of suppressing chaos. However, some literatures point out that the transient chaos and fractal basin boundary are associated with this criterion [26-29].

Through the above analysis, we denote the first-threshold value as $\mu_{1}=0.15$ for the generation of chaotic behavior in Smale horseshoe sense and the second-threshold value as $\mu_{2}=0.205$ for TLE changing from negative to positive. The attractors and transient states of system (2) are shown in Figure 7 for $\mu_{1}=0.1,0.2$, and 0.3 . When $\mu=0.1<\mu_{1}$, there are two coexisting $1 T$ periodic attractors, and the transient behaviors are nonchaotic; and when $\mu_{1}<\mu=0.2<\mu_{2}$, the attractors are still periodic, two $1 T$ attractors together with two $2 \mathrm{~T}$ attractors, but the transient state becomes chaotic. With the increasing of $\mu$, the permanent chaos first appears 


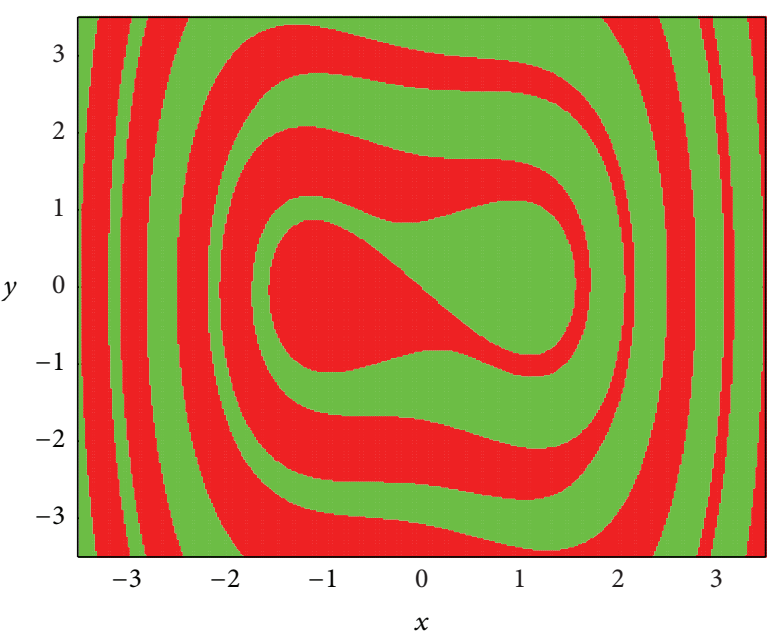

(a)

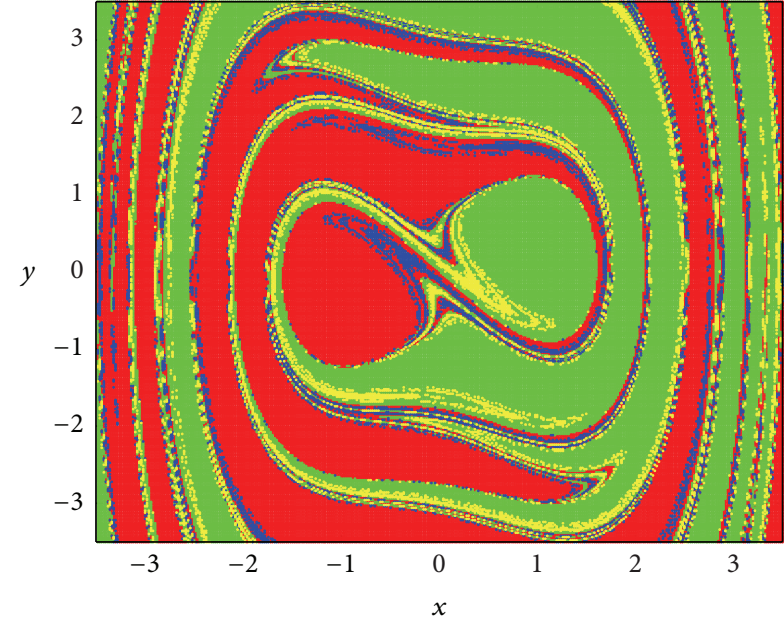

(b)

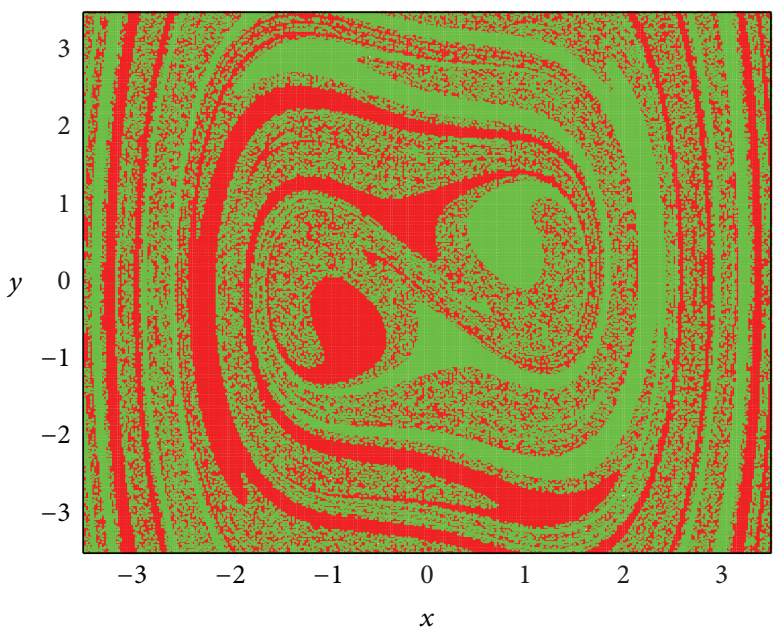

(c)

FIgURE 8: The basins of attractions for different values of $\mu$ : (a) $\mu=1.0$, (b) $\mu=2.0$, and (c) $\mu=3.0$.

at $\mu_{2}$ when TLE turns to positive as shown in Figures $1(\mathrm{~b})$ and 6 . Since then, the behaviors of system (2) begin to transform between different chaotic states, such as transient chaos in Figure 7 (c) for $\mu_{1}=0.3$ and permanent chaotic attractor in Figure $1(\mathrm{~d})$ for $\mu_{1}=0.35$. Thus, it is clear that once $\mu>$ $\mu_{1}$, the behavior of system will be either transient chaos or permanent chaos.

From the basins of attraction in Figure 8, when $\mu<\mu_{1}$, the boundary is smooth and regular accompanied with a pair of $1 T$ attractors as shown in Figure 1(a). Since $\mu>\mu_{1}$, the boundary becomes fractal even for the basins of periodic attractors as shown in Figures 8(b) and 8(c). It can be drawn that there must be chaotic saddle resulted from the existence of transient chaos and fractal boundaries. Above all, it is illustrated that once the Melnikov function has simple zero, indeed, system (2) is chaotic in sense of Smale horseshoe according to the Smale-Birkhoff theorem, in the forms of transient chaos or permanent chaos, which can be separated by checking the sign of TLE.

\section{Conclusions}

In this research, the double-well Duffing system with harmonic parametrical excitation is studied, and the aim is to examine the process and mechanism of chaotic behavior from birth to the growth. By checking the dynamics in the view of Poincaré sections and multibifurcation diagram of parameter $\mu$, we can see that there are plenty of complex dynamical behaviors, such as coexistence and symmetry of attractors, a variety of bifurcations, and different kinds of crises. In order to find the origin of chaotic motions, Melnikov's method is used. Based on the Smale-Birkhoff theorem, the critical condition for the generation of chaos in Smale horseshoe sense is explored, by calculating the sample zeros of Melnikov's function. We find that, before the appearance of chaotic attractor, the system has already been chaotic after the transversal intersection of the stable and unstable manifolds, existing as chaotic saddle. What is more, the existence of chaotic saddle also can be testified 
by the appearance of transient chaos and fractal basin boundary. Taking all the above and our previous research into consideration, consequently, this double-well Duffing system with parametrical excitation will keep on switching between chaotic saddle and permanent chaos by bifurcation and crisis, once the system becomes chaotic in sense of Smale horseshoe.

\section{Competing Interests}

The authors declare that they have no competing interests.

\section{Acknowledgments}

This research is supported by the National Natural Science Foundation of China (nos. 11302170, 11302171, and 11672232) and the Project Supported by Natural Science Basic Research Plan in Shaanxi Province of China (Program no. 2016JQ1015).

\section{References}

[1] I. Kovacic and M. J. Brennan, The Duffing Equation: Nonlinear Oscillators and Their Behaviour, John Wiley \& Sons, 2011.

[2] M. H. Matheny, M. Grau, L. G. Villanueva, R. B. Karabalin, M. C. Cross, and M. L. Roukes, "Phase synchronization of two anharmonic nanomechanical oscillators," Physical Review Letters, vol. 112, no. 1, Article ID 014101, 2014.

[3] C.-L. Chen, H.-T. Yau, C.-C. Cho, and C.-K. Chen, "Enhancement of microfluidic mixing using harmonic and chaotic electric fields," International Journal of Nonlinear Sciences and Numerical Simulation, vol. 10, no. 11-12, pp. 1545-1553, 2009.

[4] S. Li, Q. Li, J. Li, and J. Feng, "Chaos prediction and control of Goodwin's nonlinear accelerator model," Nonlinear Analysis: Real World Applications, vol. 12, no. 4, pp. 1950-1960, 2011.

[5] P. Meekangvan, A. A. Barhorst, T. D. Burton, S. Chatterjee, and L. Schovanec, "Nonlinear dynamical model and response of avian cranial kinesis," Journal of Theoretical Biology, vol. 240, no. 1, pp. 32-47, 2006.

[6] Y. Ueda, "Randomly transitional phenomena in the system governed by Duffing's equation," Journal of Statistical Physics, vol. 20, no. 2, pp. 181-196, 1979.

[7] X. L. Yue, W. Xu, and L. Wang, "Global analysis of boundary and interior crises in an elastic impact oscillator," Communications in Nonlinear Science and Numerical Simulation, vol. 18, no. 12, pp. 3567-3574, 2013.

[8] Y. Zhang, Y.-M. Lei, and T. Fang, "Symmetry breaking crisis of chaotic attractors," Acta Physica Sinica, vol. 58, no. 6, pp. 37993805, 2009.

[9] O. I. Olusola, U. E. Vincent, and A. N. Njah, "Synchronization, multistability and basin crisis in coupled pendula," Journal of Sound and Vibration, vol. 329, no. 4, pp. 443-456, 2010.

[10] E. Ott, Chaos in Dynamical Systems, Cambridge University Press, 2nd edition, 2002.

[11] Z. K. Sun, W. Xu, X. L. Yang, and T. Fang, "Inducing or suppressing chaos in a double-well Duffing oscillator by time delay feedback," Chaos, Solitons \& Fractals, vol. 27, no. 3, pp. 705-714, 2006.

[12] D. Liu, J. Li, and Y. Xu, "Principal resonance responses of SDOF systems with small fractional derivative damping under narrow-band random parametric excitation," Communications in Nonlinear Science and Numerical Simulation, vol. 19, no. 10, pp. 3642-3652, 2014.

[13] A. Syta, G. Litak, S. Lenci, and M. Scheffler, "Chaotic vibrations of the Duffing system with fractional damping," Chaos, vol. 24, no. 1, Article ID 013107, 2014.

[14] M. G. Tehrani, L. Wilmshurst, and S. J. Elliott, "Receptance method for active vibration control of a nonlinear system," Journal of Sound and Vibration, vol. 332, no. 19, pp. 4440-4449, 2013.

[15] X. R. Zhao, W. Xu, Y. G. Yang, and X. Y. Wang, "Stochastic responses of a viscoelastic-impact system under additive and multiplicative random excitations," Communications in Nonlinear Science and Numerical Simulation, vol. 35, pp. 166-176, 2016.

[16] Y. Zhang, B. Rossetto, W. Xu, X. Yue, and T. Fang, "Roles of chaotic saddle and basin of attraction in bifurcation and crisis analysis," International Journal of Bifurcation and Chaos, vol. 21, no. 3, pp. 903-915, 2011.

[17] Y. Zhang, L. Du, X. L. Yue, Q. Han, and T. Fang, "Analysis of symmetry breaking bifurcation in duffing system with random parameter," Computer Modeling in Engineering \& Sciences, vol. 106, no. 1, pp. 37-51, 2015.

[18] V. K. Melnikov, "On the stability of a center for time-periodic perturbations," Transactions of the Moscow Mathematical Society, vol. 12, pp. 1-57, 1963.

[19] J. Guckenheimer and P. Holmes, Nonlinear Oscillations, Dynamical Systems, and Bifurcations of Vector Fields, Springer, New York, NY, USA, 1983.

[20] P. J. Holmes, "A nonlinear oscillator with a strange attractor," Philosophical Transactions of the Royal Society of London, Series A: Mathematical and Physical Sciences, vol. 292, no. 1394, pp. 419-448, 1979.

[21] S. Wiggins, Global Bifurcations and Chaos: Analytical Methods, vol. 73 of Applied Mathematical Sciences, Springer, New York, NY, USA, 1988.

[22] S. Wiggins, Introduction to Applied Nonlinear Dynamical Systems and Chaos, vol. 2 of Texts in Applied Mathematics, Springer, New York, NY, USA, 1990.

[23] J. G. Margallo and J. D. Bejarano, "Melnikov's method for nonlinear oscillators with non-linear excitations," Journal of Sound and Vibration, vol. 212, no. 2, pp. 311-319, 1998.

[24] A. Wolf, J. B. Swift, H. L. Swinney, and J. A. Vastano, "Determining Lyapunov exponents from a time series," Physica D: Nonlinear Phenomena, vol. 16, no. 3, pp. 285-317, 1985.

[25] A. Wolf and T. Bessoir, "Diagnosing chaos in the space circle," Physica D: Nonlinear Phenomena, vol. 50, no. 2, pp. 239-258, 1991.

[26] F. C. Moon and G. X. Li, "Fractal basin boundaries and homoclinic orbits for periodic motion in a two-well potential," Physical Review Letters, vol. 55, no. 14, pp. 1439-1442, 1985.

[27] R. Chacón and J. D. Bejarano, "Homoclinic and heteroclinic chaos in a triple-well oscillator," Journal of Sound and Vibration, vol. 186, no. 2, pp. 269-278, 1995.

[28] M. S. Siewe, H. Cao, and M. A. F. Sanjuán, "Effect of nonlinear dissipation on the basin boundaries of a driven two-well Rayleigh-Duffing oscillator," Chaos, Solitons and Fractals, vol. 39, no. 3, pp. 1092-1099, 2009.

[29] M. S. Siewe, C. Tchawoua, and P. Woafo, "Melnikov chaos in a periodically driven Rayleigh-Duffing oscillator," Mechanics Research Communications, vol. 37, no. 4, pp. 363-368, 2010. 


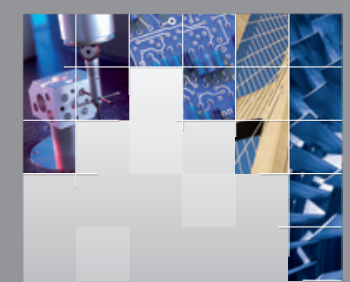

\section{Enfincering}
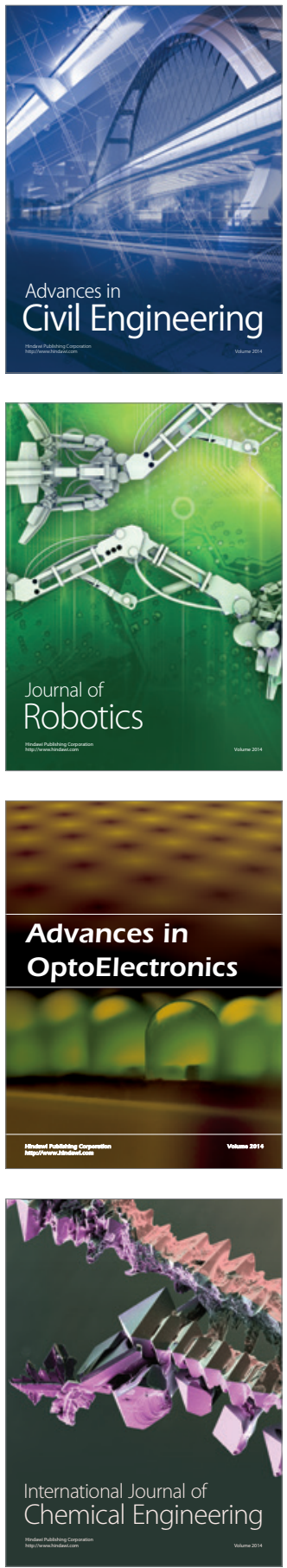

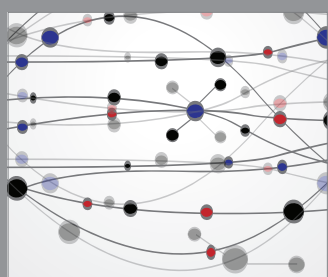

The Scientific World Journal

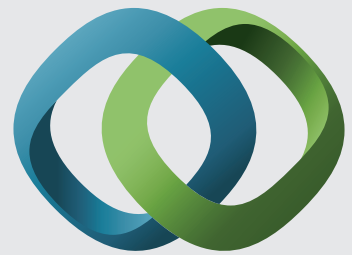

\section{Hindawi}

Submit your manuscripts at

http://www.hindawi.com
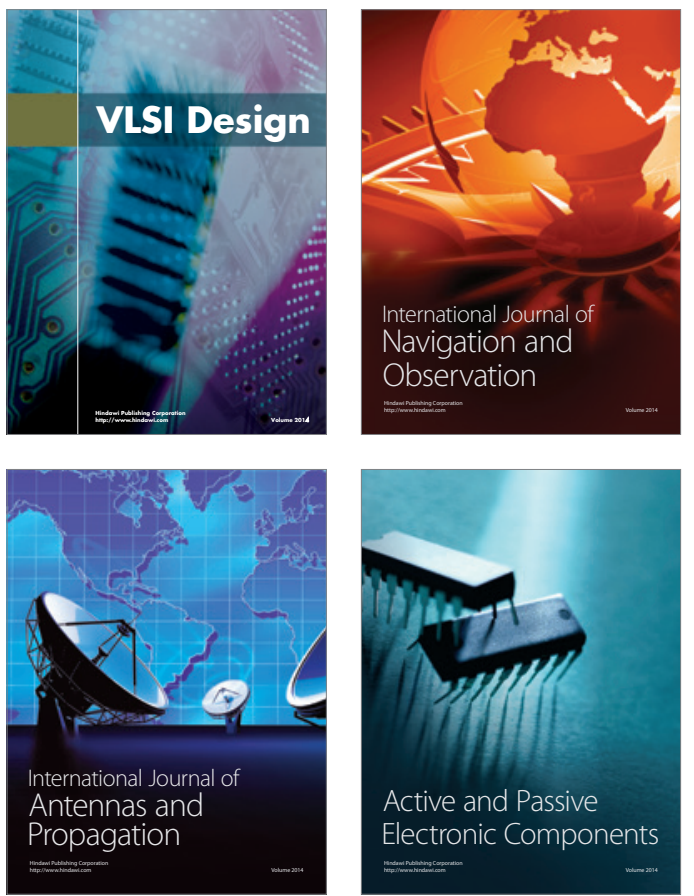
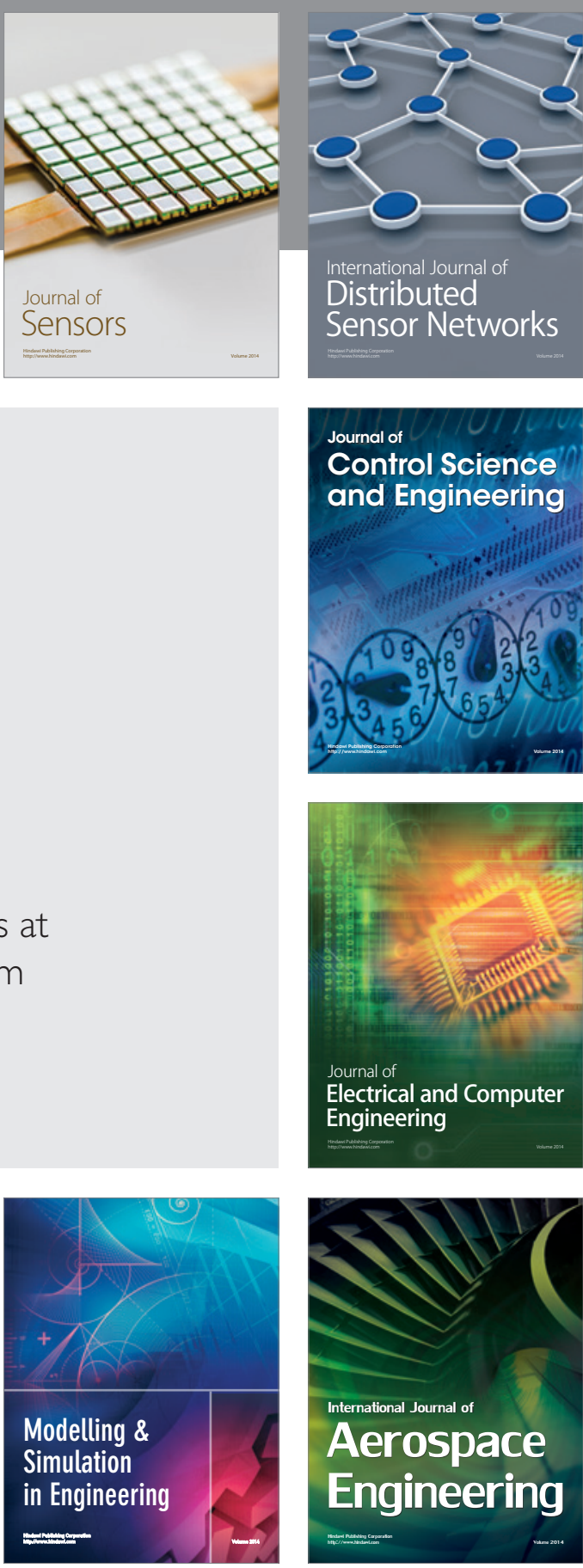

International Journal of

Distributed

Sensor Networks

Journal of

Control Science

and Engineering
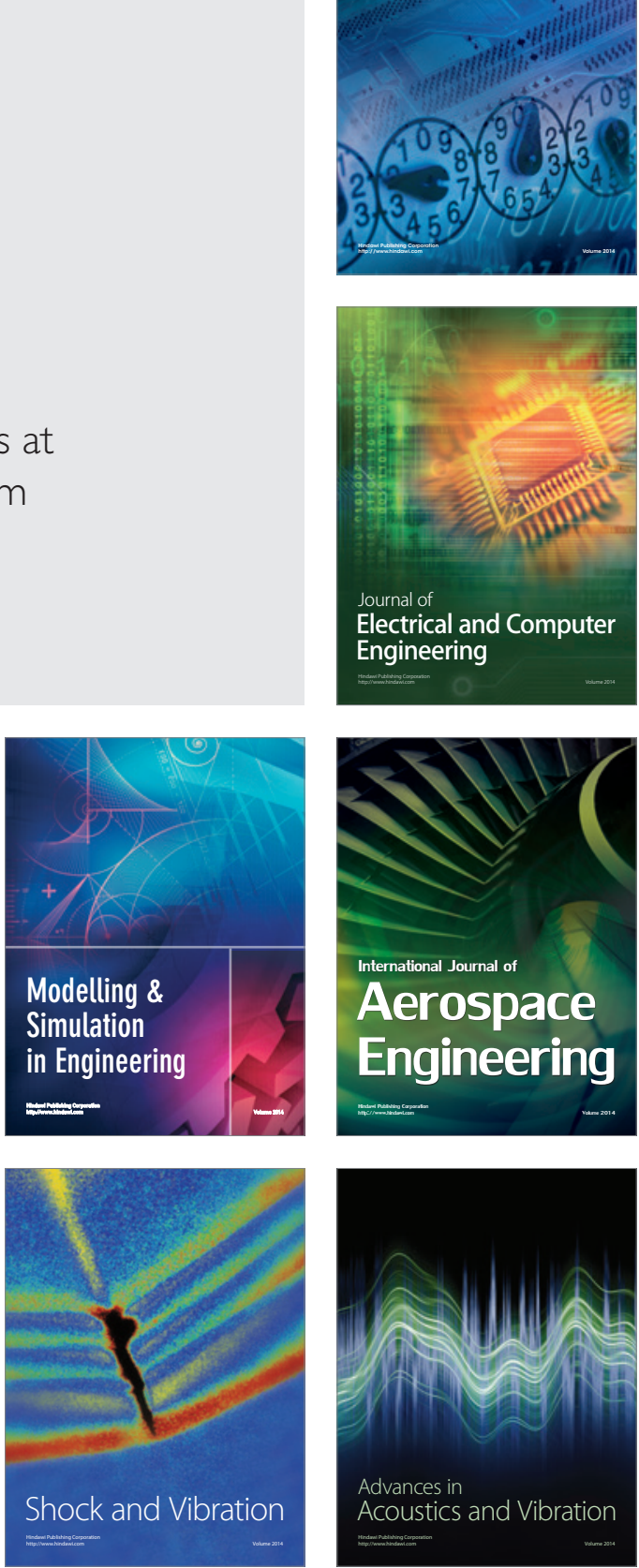